\title{
Acknowledgement to reviewers 2012
}

Published online: 17 March 2013

(C) Springer-Verlag Berlin Heidelberg 2013

The Editors and publisher gratefully acknowledge the excellent work performed by our reviewers in the year 2012. The quality of Oral and Maxillofacial Surgery is definitely depending on the review process. The work laid down by our reviewers is essential for an increasing interest of our journal in Europe and outside.

We thank all the reviewers for their excellent expertise which helps to increase the reputation of the journal. Without their dedicated hard and time-consuming work such achievement would not be possible.

The Editorial Office as well all authors and certainly also the broad readership of the Journal express their sincere gratitude to all reviewers!

Friedrich W. Neukam

Peter Ward Booth

(Editors-in-Chief)

\author{
Al-Nawas, Bilal \\ Barrett, Bill \\ Bisase, Brian \\ Bordbar, Patrishia \\ Brennan, Peter \\ Breuer, Georg \\ Cheung, Lim \\ Coombes, Darryl \\ Curran, Joy \\ Devlin, Mark \\ Eckelt, Uwe \\ Eckert, Alexander \\ Edwards, Barry \\ Eitner, Stephan \\ Fan, Kathleen \\ Finlay, Patricia \\ Francis, Ian \\ Freier, Kolja \\ Fromm, Martin \\ Gaggl, Alexander \\ Gellrich, Nils-Claudius \\ Gundlach, Karsten K. H. \\ Haßfeld, Stefan
}

Hemprich, Alexander
Hochban, Walter
Hoffmann, Jürgen
Holland, Ian
Holmes, Simon
Huppa, Christoph
Kleinheinz, Johannes
Kübler, Alexander
Lauer, Günter
Lell, Michael
Loukota, Richard
Metelmann, Hans-Robert
Müller, Fabian
Neff, Andreas
Nkenke, Emeka
Palmer, Richard
Precious, David
Radespiel-Tröger, Martin
Reich, Rudolf
Reinert, Siegmar
Rücker, Martin
Schendel, Stephen
Schlegel, Andreas

Schliephake, Henning

Schmelzeisen, Rainer

Schubert, Johannes

Schultze-Mosgau, Stefan

Schuster, Maria

Schwarz, Frank

Schwarz-Furlan, Stephan

Sieg, Peter

Slootweg, Pieter

Springer, Ingo

St. Pierre, Michael

Stockmann, Philipp

Terheyden, Hendrik

Vairaktaris, Eleftherios

van der Waal, Isaac

Van Sickels, Joseph

Wiltfang, Jörg

Wolff, Klaus-Dietrich

Wright, Natasha

Zenk, Johannes

Zolk, Oliver 